Pacific

Journal of

Mathematics

AN ANALOGUE OF BRUMER'S CRITERION FOR

HYPERCOHOMOLOGY GROUPS AND CLASS FORMATIONS

YOSHIHIRO KOYA 


\title{
AN ANALOGUE OF BRUMER'S CRITERION FOR HYPERCOHOMOLOGY GROUPS AND CLASS FORMATIONS
}

\author{
YOSHIHIRO KOYA
}

\begin{abstract}
We generalize to hypercohomology the Brumer criterion for the strict cohomological dimension of profinite groups.
\end{abstract}

\section{Introduction}

The aim of this paper is to obtain a criterion determining the strict cohomological dimension of a profinite group.

The strict cohomological dimension of a profinite group $G$, written $\operatorname{scd}_{p} G$, is the smallest integer $n$ such that the $p$-primary component of $H^{n+1}(G, M)$ vanishes for all discrete $G$-modules $M$, where $p$ is a prime number. There is also the notion of cohomological dimension of $G$, denoted by $\operatorname{cd}_{p} G$. This is the smallest integer $n$ such that $H^{n+1}(G, A)=0$ for all discrete $p$-primary $G$-modules $A$.

It is well-known that the strict cohomological dimension of $G$ is equal to $\operatorname{cd}_{p} G$ or $\operatorname{cd}_{p} G+1$. This result is often useful. In many cases, in fact, it enables us to obtain sufficient information on the cohomology groups of a given profinite group. It is, nevertheless, quite difficult to determine the strict cohomological dimension of a given profinite group. Brumer gave a useful criterion determining it [1]:

Theorem 1.1 (Theorem $6.1,[\mathbf{1}]$ ). The following are equivalent for a class formation $(G, A)$ :

(1) $\operatorname{scd}_{p} G=2$.

(2) For each integer $q$ and each pair $H \subset K$ of open subgroups of $G$ such that $H$ is normal in $K$, the homomorphism induced by the reciprocity map

$$
\widehat{H}^{q}\left(K / H, A^{H}\right) \rightarrow \widehat{H}^{q}\left(K / H, H^{\mathrm{ab}}\right)
$$

induces an isomorphism onto on the p-primary component of the respective cohomology groups.

Using this criterion, Brumer showed, for example, that the absolute Galois group of a local field has strict cohomological dimension two.

The author developed in [8] the theory of class formations, including complexes and hypercohomology groups. Class formations can be applied 
to higher-dimensional local fields and, of course, to the classical class field theory of usual local and global fields. Then the problem is whether one can generalize the Brumer criterion to the case of such class formations. This is the main motivation of this paper. We prove:

Theorem 1.2 (cf. Theorem 3.2). The following statements are equivalent for a class formation $\left(G, A^{\bullet}\right)$ :

(1) $\operatorname{scd}_{p} G \leq n+1$.

(2) For each integer $q$ and each pair $H \subset K$ of open subgroups of $G$ such that $H$ is normal in $K$, the homomorphisms induced by the reciprocity map induce isomorphisms

$$
\widehat{H}^{q}\left(K / H, H^{-r}\left(H, A^{\bullet}\right)\right)(p) \simeq \widehat{H}^{q}\left(K / H, H_{r+1}(H, \mathbb{Z})\right)(p)
$$

for each $r=0, \ldots, n-1$.

This is a generalization of the Brumer criterion, as can be seen by examining the case $n=1$ (note that $\operatorname{scd}_{p} G=1$ if and only if $G=\{1\}$ ).

As an application, we determine the strict cohomological dimension of a two-dimensional local field, as Brumer did. Note that the point of this paper is to obtain a necessary and sufficient condition determining the strict cohomological dimension. If one wishes to determine only the strict cohomological dimension of higher local fields, there are other methods (see Section 4).

Notation 1.3. We will freely use standard notation about complexes and hypercohomology groups. Unless the contrary is explicitly stated, we employ the following notations and conventions:

(1) For an abelian group $A$, we denote the group of $n$-torsion elements of $A$ by $A_{n}$. In particular, in the case $n$ is a prime number, $A(p)$ stands for $p$-primary torsion part of $A$.

(2) For a field $F$, we denote the separable closure of $F$ by $F_{s}$.

\section{Generalities}

In this section we obtain a criterion determining the strict cohomological dimension of a profinite group $G$ in rather general contexts.

Unless the contrary is explicitly stated, let $G$ be a profinite group, and let $H \subset K$ be open subgroups of $G$ such that $H$ is normal in $K$.

First of all, we fix notation used frequently in this paper.

Let $G$ be as above. We may assume that

$$
G=\lim _{U} G / U,
$$

where $U$ runs through open normal subgroups of $G$. We define the complete group algebra $\mathbb{Z}_{p} \llbracket G \rrbracket$ of $G$ over the ring of $p$-adic integers to be the inverse 
limit of the ordinary group ring of the finite quotients $G / U$ of $G$ over $\mathbb{Z}_{p}$ :

$$
\mathbb{Z}_{p} \llbracket G \rrbracket=\lim _{U} \mathbb{Z}_{p}[G / U] .
$$

For an arbitrary $\mathbb{Z}_{p} \llbracket G \rrbracket$-module $A$, we define the functor $F_{G}$ by

$$
F_{G}(A)=A / A I(G),
$$

where $I(G)$ is the closed ideal of $\mathbb{Z}_{p} \llbracket G \rrbracket$ generated by $\{1-g \mid g \in G\}$.

Next, consider an exact sequence

$$
\cdots P_{n} \rightarrow P_{n-1} \rightarrow \cdots \rightarrow P_{0} \rightarrow \mathbb{Z}_{p} \rightarrow 0
$$

where, for each $i, P_{i}$ is a projective $\mathbb{Z}_{p} \llbracket G \rrbracket$-module. We let $Q_{n}$ denote $\operatorname{Ker}\left(P_{n} \rightarrow P_{n-1}\right)$, and formally define $Q_{-1}$ to be $\mathbb{Z}_{p}$. Then the sequence

$$
0 \rightarrow H_{n+1}\left(H, \mathbb{Z}_{p}\right) \rightarrow F_{H}\left(Q_{n}\right) \rightarrow F_{H}\left(P_{n}\right) \rightarrow F_{H}\left(Q_{n-1}\right) \rightarrow 0
$$

is exact. From this short exact sequence we obtain an induced homomorphism

$$
\omega^{q}: \widehat{H}^{q-2}\left(K / H, F_{H}\left(Q_{n-2}\right)\right) \rightarrow \widehat{H}^{q}\left(K / H, H_{n}\left(H, \mathbb{Z}_{p}\right)\right)
$$

defined as the composition of two boundary maps.

Lemma 2.1. Let $H \subset K$ be as above and let $q$ be an arbitrary integer. The following statements are equivalent:

(1) $\widehat{H}^{q-2}\left(K / H, F_{H}\left(Q_{n-2}\right)\right) \simeq \widehat{H}^{q}\left(K / H, H_{n}\left(H, \mathbb{Z}_{p}\right)\right)$.

(2) $\widehat{H}^{q}\left(K / H, F_{H}\left(Q_{n-1}\right)\right)=0$.

This is an immediate consequence of the lemma below:

Lemma 2.2. Let $T$ be a finite group and let

$$
0 \rightarrow A \rightarrow B \stackrel{\omega}{\longrightarrow} C \rightarrow D \rightarrow 0 .
$$

be an exact sequence of T-modules; we have an induced homomorphism

$$
d_{q}: \widehat{H}^{q-2}(T, D) \rightarrow \widehat{H}^{q}(T, A)
$$

defined as the composition of two boundary maps. The following statements are equivalent:

(1) For each $q, d_{q}$ is an isomorphism on the p-primary components.

(2) For each $q, \omega^{*}: \widehat{H}^{q}(T, B) \rightarrow \widehat{H}^{q}(T, C)$ is an isomorphism on the $p$ primary components.

Lemma 2.1 enables us to prove the following:

Proposition 2.3. Assume that

$$
\omega^{q}: \widehat{H}^{q-2}\left(K / H, F_{H}\left(Q_{n-2}\right)\right) \simeq \widehat{H}^{q}\left(K / H, H_{n}\left(H, \mathbb{Z}_{p}\right)\right)
$$

for each integer $q$. Then $\operatorname{scd}_{p} G \leq n+1$. 
Proof. Form the assumptions and Lemma 2.1 we can conclude that

$$
\widehat{H}^{q}\left(K / H, F_{H}\left(Q_{n-1}\right)\right)=0 .
$$

Taking into account Lemma 6.7 of $[\mathbf{1}]$, we deduce $\mathrm{hd}_{\mathbb{Z}_{p} \llbracket G \rrbracket} Q_{n-1} \leq 1$. Therefore, we can find projective $\mathbb{Z}_{p} \llbracket G \rrbracket$-modules $P_{n+1}^{\prime}$ and $P_{n}^{\prime}$ such that

$$
0 \rightarrow P_{n+1}^{\prime} \rightarrow P_{n}^{\prime} \rightarrow Q_{n-1} \rightarrow 0
$$

is exact. Thus, we can take a projective resolution of $\mathbb{Z}_{p}$ of the form

$$
0 \rightarrow P_{n+1}^{\prime} \rightarrow P_{n}^{\prime} \rightarrow P_{n-1} \rightarrow \cdots \rightarrow P_{0} \rightarrow \mathbb{Z}_{p} \rightarrow 0
$$

and therefore $H_{n+2}\left(H, \mathbb{Z}_{p}\right)=0$. Since $H^{n+2}\left(H, \mathbb{Q}_{p} / \mathbb{Z}_{p}\right) \simeq\left(H_{n+2}\left(H, \mathbb{Z}_{p}\right)\right)^{*}$, we obtain $\operatorname{cd}_{p} G \leq n+1$ (cf. Corollary 4 to Proposition 14, Chap. I, [13]).

From the exact sequence

$$
0 \rightarrow Q_{n-1} \rightarrow P_{n-1} \rightarrow \cdots \rightarrow P_{0} \rightarrow \mathbb{Z}_{p} \rightarrow 0
$$

we obtain $\operatorname{Tor}_{n+1}^{K}\left(\mathbb{Z}_{p}, \mathbb{Z}_{p}\right)=\operatorname{Tor}_{1}^{K}\left(Q_{n-1}, \mathbb{Z}_{p}\right)$. On the other hand, the proof of [1, Lemma 6.7] shows $\operatorname{Tor}_{1}^{K}\left(Q_{n-1}, \mathbb{Z}_{p}\right)=0$. Applying [1, Corollary 5.5], we prove the proposition.

The following lemma gives us the converse of Proposition 2.3.

Lemma 2.4. Suppose that we have $\operatorname{scd}_{p} G \leq n+1$. Then we can conclude

$$
\omega^{q}: \widehat{H}^{q-2}\left(K / H, F_{H}\left(Q_{n-2}\right)\right) \simeq \widehat{H}^{q}\left(K / H, H_{n}\left(H, \mathbb{Z}_{p}\right)\right)
$$

for an arbitrary integer $q$.

Proof. Let

$$
\cdots \rightarrow P_{n} \rightarrow P_{n-1} \rightarrow \cdots \rightarrow P_{0} \rightarrow \mathbb{Z}_{p} \rightarrow 0
$$

be a projective resolution of $\mathbb{Z}_{p}$. Added to this, we denote the complex

$$
\cdots \rightarrow \underbrace{F_{H}\left(P_{n}\right)}_{\operatorname{deg}-2} \rightarrow \underbrace{F_{H}\left(P_{n-1}\right)}_{\operatorname{deg}-1} \rightarrow \underbrace{F_{H}\left(P_{n-2}\right)}_{\operatorname{deg} 0} \rightarrow 0 \rightarrow 0 \rightarrow \cdots
$$

by $C^{\bullet}$. Since the sequence

$$
\rightarrow P_{n} \rightarrow P_{n-1} \rightarrow P_{n-2} \rightarrow Q_{n-3} \rightarrow 0
$$

is exact,

$$
\tau_{\leq-1} C^{\bullet} \rightarrow C^{\bullet} \rightarrow F_{H}\left(Q_{n-3}\right) \rightarrow \tau_{\leq-1} C^{\bullet}[-1]
$$

is a distinguished triangle. For sufficiently large $q$, we obtain

$$
H_{q-1}\left(K, Q_{n-3}\right) \simeq \widehat{H}^{-q}\left(K / H, C^{\bullet}\right) .
$$

Since $\operatorname{scd}_{p} G \leq n+1$, we have $\widehat{H}^{-q}\left(K / H, C^{\bullet}\right)=0$ for sufficiently large $q$, and therefore, for all $q$, we have $\widehat{H}^{-q}\left(K / H, C^{\bullet}\right)=0$. Thus, we can conclude

$$
\widehat{H}^{q-1}\left(K / H, F_{H}\left(Q_{n-3}\right)\right) \simeq \widehat{H}^{q}\left(K / H, \tau_{\leq-1} C^{\bullet}\right),
$$


for each integer $q$.

However, the assumption $\operatorname{scd}_{p} G \leq n+1$ gives $H_{n+1}\left(H, \mathbb{Z}_{p}\right)=0$, so we know $H^{q}\left(\tau_{\leq-1} C^{\bullet}\right)=0$ for $q<-2$. That is, we have a distinguished triangle

$$
H_{n}\left(H, \mathbb{Z}_{p}\right)[2] \rightarrow \tau_{\leq-1} C^{\bullet} \rightarrow H_{n-1}\left(H, \mathbb{Z}_{p}\right)[-1] \rightarrow H_{n}\left(H, \mathbb{Z}_{p}\right)[1] .
$$

This triangle induces a long exact sequence of hypercohomology groups:

$$
\begin{aligned}
\cdots \rightarrow \widehat{H}^{q+2}\left(K / H, H_{n}\left(H, \mathbb{Z}_{p}\right)\right) & \rightarrow \widehat{H}^{q}\left(K / H, \tau_{\leq-1} C^{\bullet}\right) \\
& \rightarrow \widehat{H}^{q+1}\left(K / H, H_{n-1}\left(H, \mathbb{Z}_{p}\right)\right) \rightarrow \cdots
\end{aligned}
$$

Now consider the commutative diagram

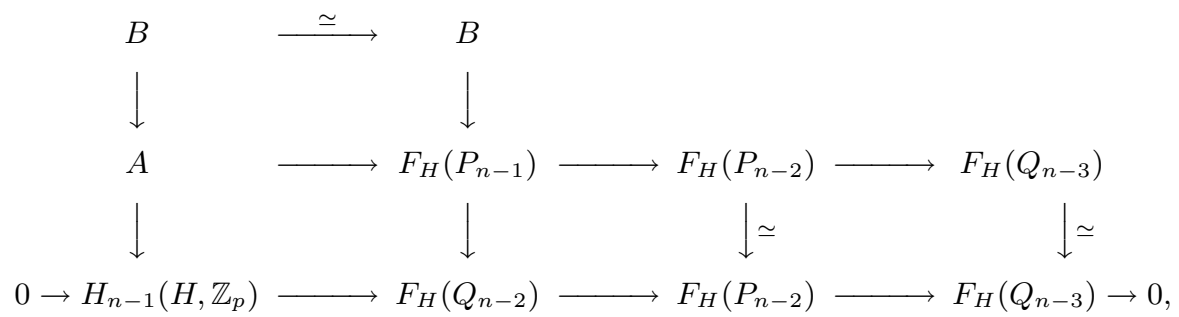

where we have put

$$
A=\operatorname{Ker}\left(F_{H}\left(P_{n-1}\right) \rightarrow F_{H}\left(P_{n-2}\right)\right), \quad B=\operatorname{Ker}\left(F_{H}\left(P_{n-1}\right) \rightarrow F_{H}\left(Q_{n-2}\right)\right) .
$$

All horizontal sequences are exact. Applying the snake lemma, we have an exact sequence

$$
\rightarrow \widehat{H}^{q+1}(K / H, B) \rightarrow \widehat{H}^{q+1}(K / H, A) \rightarrow \widehat{H}^{q+1}\left(K / H, H_{n-1}\left(H, \mathbb{Z}_{p}\right)\right) \rightarrow \cdots .
$$

By observing the middle horizontal sequence in the preceding diagram, we have

$$
\widehat{H}^{q-1}\left(K / H, F_{H}\left(Q_{n-3}\right)\right) \simeq \widehat{H}^{q+1}(K / H, A),
$$

and therefore, from (1), we also have

$$
\widehat{H}^{q+1}(K / H, A) \simeq \widehat{H}^{q}\left(K / H, \tau_{\leq-1} C^{\bullet}\right) .
$$

On the other hand, from the definition of $B$, we know that

$$
\widehat{H}^{q}\left(K / H, F_{H}\left(Q_{n-2}\right)\right) \simeq \widehat{H}^{q+1}(K / H, B) .
$$

Thus, we have an exact sequence

$$
\begin{aligned}
\cdots \rightarrow \widehat{H}^{q}\left(K / H, F_{H}\left(Q_{n-2}\right)\right) \rightarrow \widehat{H}^{q} & \left(K / H, \tau_{\leq-1} C^{\bullet}\right) \\
& \rightarrow \widehat{H}^{q+1}\left(K / H, H_{n-1}\left(H, \mathbb{Z}_{p}\right)\right) \rightarrow \cdots .
\end{aligned}
$$

By comparing this exact sequence with (2), we have

$$
\widehat{H}^{q-2}\left(K / H, F_{H}\left(Q_{n-2}\right)\right) \simeq \widehat{H}^{q}\left(K / H, H_{n}\left(H, \mathbb{Z}_{p}\right)\right) .
$$

Thus, we obtain the following criterion determining the strict cohomological dimension of $G$ : 
Theorem 2.5. The following conditions are equivalent:

(1) $\operatorname{scd}_{p} G \leq n+1$.

(2) For each $q, \widehat{H}^{q-2}\left(K / H, F_{H}\left(Q_{n-2}\right)\right) \simeq \widehat{H}^{q}\left(K / H, H_{n}\left(H, \mathbb{Z}_{p}\right)\right)$.

\section{Relation to class formations}

Let $G$ be a profinite group and $A^{\bullet}$ a complex of $G$-modules acyclic outside $[-n, 0]$. Assume that the pair $\left(G, A^{\bullet}\right)$ forms a class formation. See [8] about such class formation including complexes. In this case, as in [1], we can make more explicit the conditions to determine $\operatorname{sed}_{p} G$.

Lemma 3.1. Assume that the pair $\left(G, A^{\bullet}\right)$ forms a class formation, where $A^{\bullet}$ is acyclic outside $[-n, 0]$. The following conditions are equivalent:

(1) For each $r=0, \ldots, n-1$,

$$
\widehat{H}^{q}\left(K / H, H^{-r}\left(H, A^{\bullet}\right)\right)(p) \simeq \widehat{H}^{q}\left(K / H, H_{r+1}(H, \mathbb{Z})\right)(p) .
$$

(2) For each $q, \widehat{H}^{q-2}\left(K / H, F_{H}\left(Q_{n-2}\right)\right) \simeq \widehat{H}^{q}\left(K / H, H_{n}\left(H, \mathbb{Z}_{p}\right)\right)$.

Proof. From the Tate-Nakayama theorem for hypercohomology [8], we have

$$
\widehat{H}^{q}\left(K / H, \tau_{\leq 0} R \Gamma\left(H, A^{\bullet}\right)\right) \simeq \widehat{H}^{q-2}(K / H, \mathbb{Z}) .
$$

From the exact sequence

$$
0 \rightarrow H_{1}\left(H, \mathbb{Z}_{p}\right) \rightarrow F_{H}\left(Q_{0}\right) \rightarrow F_{H}\left(P_{0}\right) \rightarrow \mathbb{Z}_{p} \rightarrow 0
$$

we obtain the following long exact sequence of cohomology groups:

$$
\begin{aligned}
\cdots \rightarrow \widehat{H}^{q-2}\left(K / H, \mathbb{Z}_{p}\right) & \rightarrow \widehat{H}^{q}\left(K / H, H_{1}\left(H, \mathbb{Z}_{p}\right)\right) \\
& \rightarrow \widehat{H}^{q}\left(K / H, F_{H}\left(Q_{0}\right)\right) \rightarrow \widehat{H}^{q-1}\left(K / H, \mathbb{Z}_{p}\right) \rightarrow \cdots
\end{aligned}
$$

Noting the results of the Tate-Nakayama theorem and the assumptions, we derive the long exact sequence

$$
\begin{aligned}
\cdots \rightarrow \widehat{H}^{q}\left(K / H, \tau_{\leq 0} R \Gamma\left(H, A^{\bullet}\right)\right) & \rightarrow \widehat{H}^{q}\left(K / H, H^{0}\left(H, A^{\bullet}\right)\right) \\
\rightarrow \widehat{H}^{q}\left(K / H, F_{H}\left(Q_{0}\right)\right) & \rightarrow \widehat{H}^{q+1}\left(K / H, \tau_{\leq 0} R \Gamma\left(H, A^{\bullet}\right)\right) \rightarrow \cdots .
\end{aligned}
$$

By comparing the long exact sequence obtained from the distinguished triangle

$$
\tau_{\leq-1} R \Gamma\left(H, A^{\bullet}\right) \rightarrow \tau_{\leq 0} R \Gamma\left(H, A^{\bullet}\right) \rightarrow H^{0}\left(H, A^{\bullet}\right) \rightarrow \tau_{\leq-1} R \Gamma\left(H, A^{\bullet}\right)[-1],
$$

we can conclude that

$$
\widehat{H}^{q-2}\left(K / H, F_{H}\left(Q_{0}\right)\right) \simeq \widehat{H}^{q}\left(K / H, \tau_{\leq-1} R \Gamma\left(H, A^{\bullet}\right)\right) .
$$

Similarly we have

$$
\widehat{H}^{q-2}\left(K / H, F_{H}\left(Q_{r}\right)\right) \simeq \widehat{H}^{q}\left(K / H, \tau_{\leq-r-1} R \Gamma\left(H, A^{\bullet}\right)\right) .
$$


Therefore,

$$
\begin{aligned}
\widehat{H}^{q-2}\left(K / H, F_{H}\left(Q_{n-2}\right)\right) & \simeq \widehat{H}^{q}\left(K / H, \tau_{\leq-n+1} R \Gamma\left(H, A^{\bullet}\right)\right) \\
& \simeq \widehat{H}^{q}\left(K / H, H^{-n+1}\left(H, A^{\bullet}\right)\right) \\
& \simeq \widehat{H}^{q}\left(K / H, H_{n}\left(H, \mathbb{Z}_{p}\right)\right) .
\end{aligned}
$$

The converse can be proven in a similar manner.

From the above lemma, we have:

Theorem 3.2. The following are equivalent:

(1) For each $r=0, \ldots, n-1$,

$$
\widehat{H}^{q}\left(K / H, H^{-r}\left(H, A^{\bullet}\right)\right)(p) \simeq \widehat{H}^{q}\left(K / H, H_{r+1}(H, \mathbb{Z})\right)(p) .
$$

(2) $\operatorname{scd}_{p} G \leq n+1$.

\section{An example}

We now apply the result of the previous section to higher-dimensional local fields.

Let $K$ be a two-dimensional local field (a complete discrete valuation field whose residue field is a usual local field). Let $\mathbb{Z}(2)$ be the Lichtenbaum complex for $K$. (For basic properties and proofs, see [10], [11] and [12].) We know that the pair $\left(\operatorname{Gal}\left(K_{s} / K\right), \mathbb{Z}(2)[2]\right)$ forms a class formation [8].

We will use the criterion established in the previous section to prove the following:

Theorem 4.1. Let $K$ be an 2-dimensional local field and $p$ a prime number different from the characteristic of $K$. Then $\operatorname{scd}_{p} \operatorname{Gal}\left(K_{s} / K\right)=3$.

Remark 4.2. Tate $[\mathbf{1 4}]$ proved this for the case that $K$ is a usual local field. Brumer gave a new proof in [1, Theorem 6.1].

For general higher-dimensional local fields, we gave a proof in [9], showing the following:

Theorem 4.3 ([9]). Let $K$ be an $N$-dimensional local field and let $p$ be a prime number distinct from the characteristic of $K$. Then $\operatorname{scd}_{p} \operatorname{Gal}\left(K_{s} / K\right)=$ $N+1$.

In [9], we showed a kind of generalization of the Tate duality of Galois cohomology of higher-dimensional local fields with a finite coefficient, and determined the strict cohomological dimension of them as its application.

We have learned from K. Kato that there is a proof based on the duality of $p$-adic étale cohomology, which seems to be easier to understand and straightforward (The method given by Kato is rather different from ours). We do not know who first gave the proof mentioned by Kato. 
In order to show Theorem 4.1, we may prove the following:

Proposition 4.4. Let $L / K$ be an arbitrary finite extension of $K$. The kernel and cokernel of the homomorphisms

$$
\omega_{q}^{*}: H^{3-q}(L, \mathbb{Z}(2)) \rightarrow H_{q}(L, \mathbb{Z})
$$

are uniquely divisible for $q=1,2$. Here the maps $\omega_{q}^{*}$ are those induced from the reciprocity map of two-dimensional local class field theory.

Then, from Theorem 3.2, we can conclude $\operatorname{scd}_{p} \operatorname{Gal}\left(K_{s} / K\right) \leq 3$. On the other hand, from [4] and [5], we already $\operatorname{know} \operatorname{cd}_{p} \operatorname{Gal}\left(K_{s} / K\right)=3$. Therefore, we can deduce that the strict cohomological dimension of a twodimensional local field is 3 .

The rest of the section is devoted to the proof of the proposition. We need the following lemma, an easy consequence of [7, Chap. I, §2, Theorem 2].

Lemma 4.5. Let $K$ be a 2-dimensional local filed and $p$ a prime number. Suppose that $K$ contains a primitive $p$-th root of unity. Then the canonical homomorphism

$$
H^{r}\left(K, \mu_{p}^{\otimes 2}\right) \rightarrow H_{3-r}(K, \mathbb{Z} / p \mathbb{Z})
$$

is bijective for $r=0,1,2$.

By using the standard arguments on the restriction and the corestriction maps for the Galois cohomology, we may assume that the field $L$ contains a primitive $p$-th root of unity.

First we prove $\operatorname{Ker} \omega_{2}^{*}=\operatorname{Coker} \omega_{2}^{*}=0$.

Consider the commutative diagram

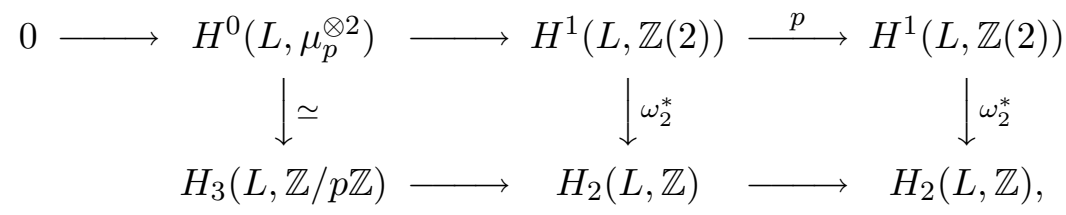

where the upper and lower horizontal sequences are both exact. From this diagram, Ker $\omega_{2}^{*}$ is $p$-torsion-free. Since the group Ker $\omega_{2}^{*}$ is a torsion group, $\operatorname{Ker} \omega_{2}^{*}=0$. The homomorphism $H_{3}(L, \mathbb{Z} / p \mathbb{Z}) \rightarrow H_{2}(L, \mathbb{Z})$ in the diagram is also injective. Thus, we may conclude Coker $\omega_{2}^{*}$ is $p$-torsion-free, and therefore, we have Coker $\omega_{2}^{*}=0$. (The group $H_{2}(L, \mathbb{Z})$ is also a torsion group.)

Next we prove that the group Ker $\omega_{1}^{*}$ is uniquely $p$-divisible. Consider the commutative diagram

0

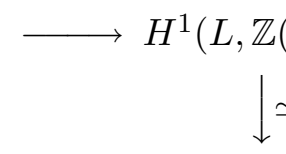

0

$H_{2}(L, \mathbb{Z}) / p$
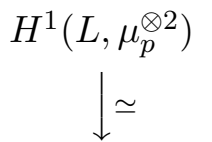

$H_{2}(L, \mathbb{Z} / p \mathbb{Z})$
$K_{2} K_{p}$

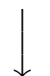

$H_{1}(L, \mathbb{Z})_{p}$ 0. 
Here the fact that the left vertical arrow is an isomorphism can be deduced from the vanishing of $\operatorname{Ker} \omega_{2}^{*}$ and Coker $\omega_{2}^{*}$. From an investigation of the preceding diagram we see that the map $K_{2} K_{p} \rightarrow H_{1}(L, \mathbb{Z})_{p}$ is an isomorphism. On the other hand, by considering the commutative diagram

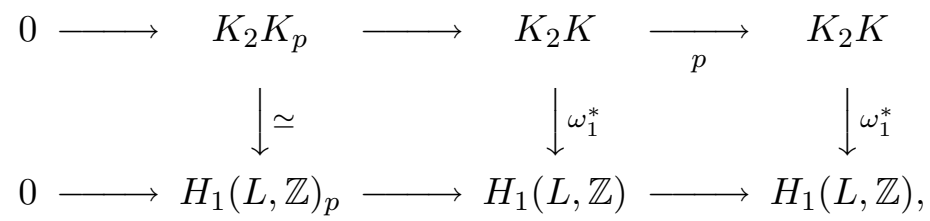

we can conclude that the groups Ker $\omega_{1}^{*}$ and Coker $\omega_{1}^{*}$ are $p$-torsion-free.

The divisibility of Ker $\omega_{1}^{*}$ can be deduced from [2, $\S 2.6$, Corollary].

Finally, the divisibility of the group Coker $\omega_{1}^{*}$ can be deduced from Lemma 4.5 .

\section{References}

[1] A. Brumer, Pseudocompact algebras, profinite groups and class formations, J. Algebra, 4 (1966), 442-470, MR 0202790 (34 \#2650), Zbl 0146.04702.

[2] I.B. Fesenko, Abelian local p-class field theory, Math. Ann., 301 (1995), 561-586, MR 1324527 (96b:11154), Zbl 0819.11057.

[3] I.B. Fesenko, Abelian extensions of complete discrete valuation fields, in 'Number theory (Paris 1993-1994)', London Math. Soc. Lect. Note Ser., 235, Cambridge Univ. Press, Cambridge 1996, 47-74, MR 1628793 (99d:11128), Zbl 0918.11061.

[4] K. Kato, A generalization of local class field theory by using K-groups I, J. Fac. Sci. Univ. Tokyo, Sect. IA, 26 (1979), 303-376, MR 0550688 (81b:12016), Zbl 0428.12013.

[5] K. Kato, A generalization of local class field theory by using K-groups II, J. Fac. Sci. Univ. Tokyo, Sect. IA, 27 (1980), 603-683, MR 0603953 (83g:12020a), Zbl 0463.12006.

[6] K. Kato, Galois cohomology of complete discrete valuation fields, Algebraic $K$-theory, Part II (Oberwolfach, 1980), Lect. Note in Math., 967, Springer-Verlag, 1982, 215238, MR 0689394 (84k:12006), Zbl 0506.12022.

[7] K. Kato and S. Saito, Two dimensional class field theory, Galois groups and their representations (Nagoya, 1981), 103-152, Adv. Stud. Pure Math., 2, North-Holland, Amsterdam, 1983, MR 0732466 (87a:11060), Zbl 0544.12011.

[8] Y. Koya, A generalization of class formation by using hypercohomology, Invent. Math., 101 (1990), 705-715, MR 1062802 (91i:11168), Zbl 0751.11055.

[9] Y. Koya, The strict cohomological dimension of higher dimensional local fields, preprint, January, 1999.

[10] S. Lichtenbaum, Values of zeta functions at nonnegative integers, Number theory, Noordwijkerhout 1983 (Noordwijkerhout, 1983), Lect. Note in Math., 1068, SpringerVerlag, Berlin-Heidelberg-New York, 1984, 127-138, MR 0756089, Zbl 0591.14014.

[11] S. Lichtenbaum, The construction of weight-two arithmetic cohomology, Invent. Math., 88 (1987), 183-215, MR 0877012 (88d:14011), Zbl 0615.14004. 
[12] S. Lichtenbaum, New results on weight-two motivic cohomology, in 'The Grothendieck Festschrift, Vol. III', Progress in Math., 88, Birkhäuser 1990, Boston-Basel-Berlin, 35-55, MR 1106910 (92m:14030), Zbl 0809.14004.

[13] J.-P. Serre, Cohomologie Galoisienne, 4th edition, 2nd corrected printing, Lect. Note in Math., 5, Springer-Verlag, 1973, MR 0404227 (53 \#8030), Zbl 0259.12011.

[14] J. Tate, Duality theorems in Galois cohomology in 'Proceedings of the International Congress of Mathematics' (Stockholm 1962), Inst. Mittag-Leffler, Djursholm, 1963, 288-295, MR 0175892 (31 \#168), Zbl 0126.07002.

Received January 20, 2004.

Department of Mathematical Sciences

Yokohama City University

22-2 SETO, KanAZAWA-KU

YOKOHAMA 236-0027

JAPAN

E-mail address: koya@yokohama-cu.ac.jp 